\title{
Vibrio vulnificus infection: a persistent threat to public health
}

\author{
Na Ra Yun and Dong-Min Kim
}

Division of Infectious Disease, Department of Internal Medicine, Chosun University College of Medicine, Gwangju, Korea

Received: May 2, 2018

Accepted: May 18, 2018

\section{Correspondence to}

Dong-Min Kim, M.D.

Division of Infectious Disease, Department of Internal Medicine, Chosun University College of Medicine, 365 Pilmun-daero, Dong-gu, Gwangju 61453, Korea Tel: $+82-62-220-3108$

Fax: +82-62-234-9653

E-mail: drongkim@chosun.ac.kr
Vibrio vulnificus is a gram-negative bacterium that can cause serious, potentially fatal infections. V. vulnificus causes three distinct syndromes: an overwhelming primary septicemia caused by consuming contaminated seafood, wound infections acquired when an open wound is exposed to contaminated warm seawater, and gastrointestinal tract-limited infections. Case-fatality rates are higher than $50 \%$ for primary septicemia, and death typically occurs within 72 hours of hospitalization. Risk factors for V. vulnificus infection include chronic liver disease, alcoholism, and hematological disorders. When V. vulnificus infection is suspected, appropriate antibiotic treatment and surgical interventions should be performed immediately. Third-generation cephalosporin with doxycycline, or quinolone with or without third-generation cephalosporin, may be potential treatment options for patients with V. vulnificus infection.

Keywords: Vibrio vulnificus; Diagnosis; Therapeutics; Prognosis

\section{INTRODUCTION}

Vibrio vulnificus is a halophilic, gram-negative bacterium belonging to the Vibrio genus and Vibrionaceae family. Other members of this family include Vibrio cholera and Vibrio parahaemolyticus, both of which cause acute gastrointestinal symptoms. However, unlike other species of this family, V. vulnificus can cause serious and fatal infections [1,2], such as gastroenteritis, skin and soft tissue infections, and primary sepsis that may progress to necrotizing fasciitis and death $[1,2]$.

\section{EPIDEMIOLOGY}

V. vulnificus is a potentially fatal foodborne pathogen $[2,3]$. The Centers for Disease Control and Prevention (CDC) estimate that the average annual incidence of all V. vunlificus infections increased by $41 \%$ between 1996 and 2005 in the United States [2,3]. In Taiwan, the number of cases attributed to V. vulnificus has increased since 1985, when the first case was reported [4].

V. vulnificus infection in Korea peaks in September and is prevalent between June and November every year (Fig. 1), the period during which seawater temperature increases. Most cases occur in areas close to the coastline in South Jeolla and South Gyeongsang provinces. This infection is most prevalent when the seawater temperature is $\geq 18^{\circ} \mathrm{C}$ to $20^{\circ} \mathrm{C}$ and the salinity is approximately $25 \%$.

V. vulnificus can be transmitted through two channels: one is the consumption of contaminated fish and shellfish, and the other is skin exposure to contaminated seawater [5]. The sensitivity of the host is crucial for the development of V. vulnificus infection, which commonly occurs in patients with chronic liver diseases, such as alcoholic liver disease, liver cirrhosis, and chronic hepatitis B or C, or who suffer from alcohol abuse without documented liver disease. Moreover, patients with chronic diseases such as diabetes mellitus, end-stage re- 


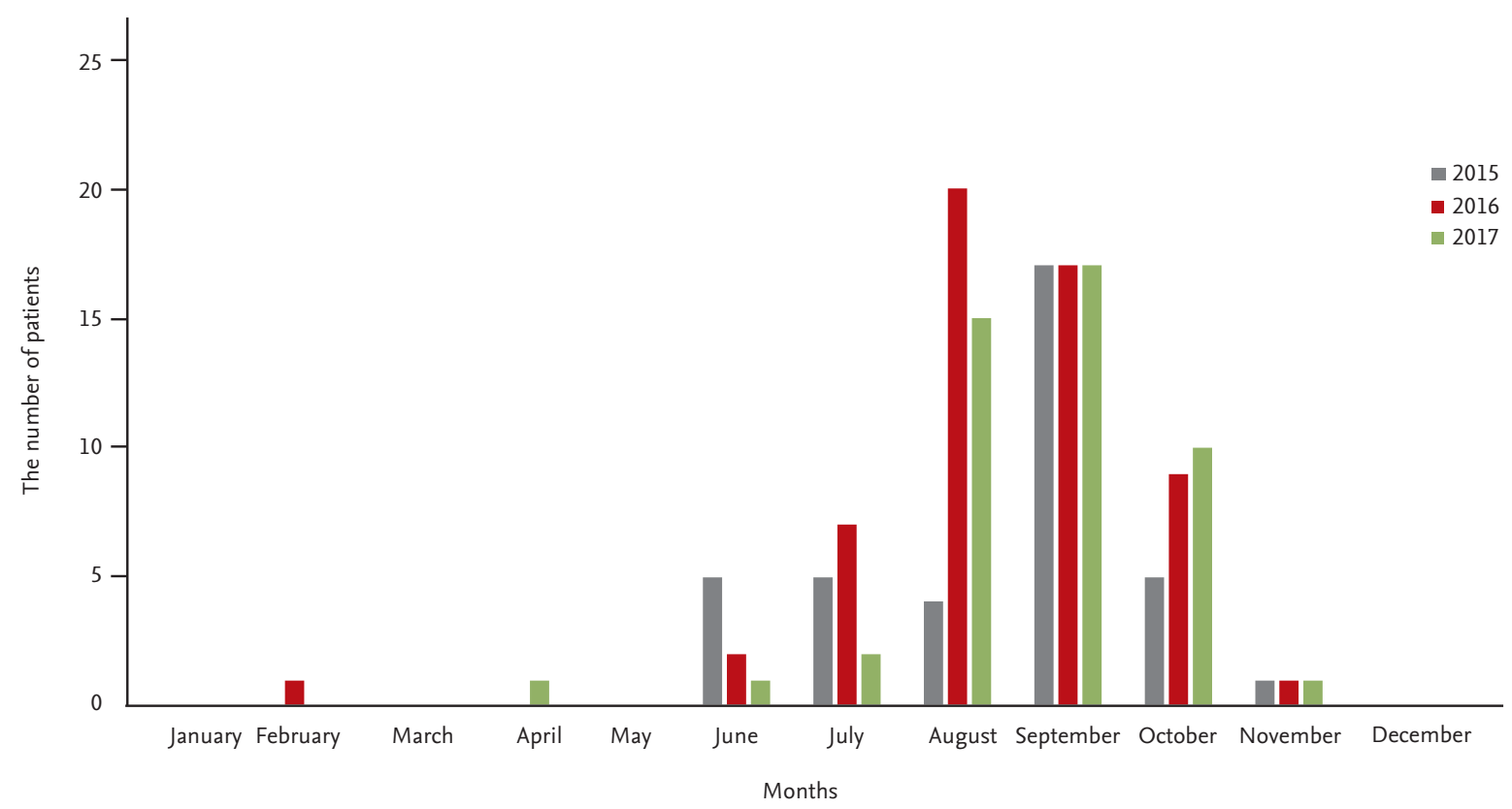

Figure 1. Monthly record on the number of patients with Vibrio vulnificus infections from 2015 to 2017 in South Korea.

nal disease, rheumatoid arthritis, and hemochromatosis are at high risk for serious V. vulnificus infection $[6,7]$.

Cirrhotic patients often have immune system dysfunction, decreased complement levels and reduced phagocytic and chemotaxis capacity; therefore, the virulence of V. vulnificus is difficult to counter in these patients $[5,8]$. Furthermore, V. vulnificus may directly enter the portal system, bypassing the hepatic reticuloendothelial system, in patients with portal hypertension $[5,8]$. According to a report on V. vulnificus sepsis in Korea, > $90 \%$ of patients with $\mathrm{V}$. vulnificus sepsis had some form of chronic disease, which included $66 \%$ with liver disease ( $43 \%$ with liver cirrhosis) and $75 \%$ with alcoholism (at least 1 oz of alcoholic beverage consumed every day) [9].

Iron levels are frequently elevated in patients with chronic liver disease and hemochromatosis, which creates a predisposition to invasive V. vulnificus infections, because this microorganism uses iron stores for bacterial overgrowth $[5,8]$. Wright et al. [10] reported that V. vulnificus cannot proliferate in normal human serum; they directly correlated its virulence with host iron availability based on their observation that iron injection in mouse models resulted in a reduction in 50\% lethal dose. Therefore, patients with hemochromatosis are known to be susceptible to V. vulnificus infection. Some cases of V. vulnificus sepsis occurred in patients with hemochromatosis. Deferoxamine, a standard iron chelation agent widely used in treating patients with iron overload, is a type of hydroxamate siderophore of the Streptomyces species; in vitro experiments have reported that deferoxamine facilitates the proliferation of ferophilic organisms, such as V. vulnificus, Yersinia enterocolitica, and members of the order Mucorales [11]. In contrast, administration of other iron-chelation agents that are not siderophores, such as deferiprone or deferasirox, is reported to have an inhibitory effect on the growth of V. vulnificus [12]. Therefore, when V. vulnificus sepsis occurs in a patient with hemochromatosis who may require iron chelation, using deferiprone or deferasirox may be more appropriate than deferoxamine.

Interestingly, men appear to have a much higher risk for serious infection than women [13,14]. In South Korea, 506 and 82 out of 588 V. vulnificus infection cases were men (86.1\%) and women (13.9\%), respectively, from 2001 to 2010 [13]. In a study in Taiwan, the rates of $V$. vulnificus infection were also higher in men than women [4]. The reason behind this difference in susceptibility is unclear. A study involving rats showed that after the administration of $\mathrm{V}$. vulnificus lipopolysaccharide extracts, the 24 -hour mortality rate was $82 \%$ in male rats and $21 \%$ in female rats. However, the mortality rate of female rats without ovaries increased to nearly $75 \%$; 


\section{$\mathrm{KJIM}^{+}$}
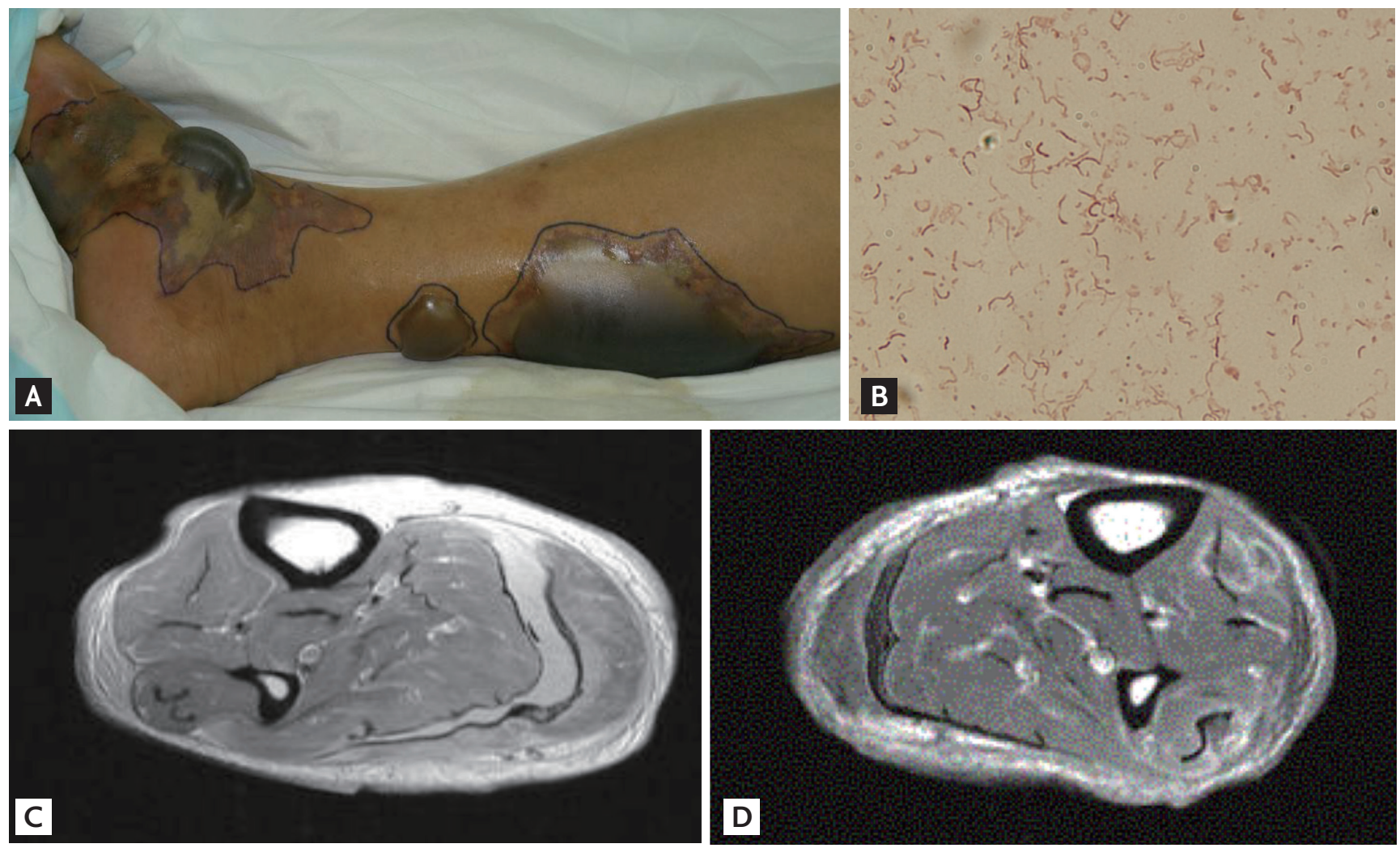

Figure 2. Characteristic lesions of patients with Vibrio vulnificus infection. (A) Characteristic skin lesions of V. vulnificus infection presenting as hemorrhagic and serous bullae; ecchymoses on the lower leg. (B) Gram stain for specimens obtained from bullae of a patient with V. vulnificus infection. Gram-negative curved bacilli isolated. (C, D) Magnetic resonance imaging for necrotizing fasciitis on the lower leg of a patient with V. vulnificus infection.

subsequent estrogen administration reduced the mortality rate to $38 \%$. This demonstrates that estrogen has protective effects against V. vulnificus sepsis [15].

\section{CLINICAL MANIFESTATIONS}

V. vulnificus causes three distinct syndromes: gastrointestinal tract limited infections, wound infections, and primary septicemia $[5,16]$. Septicemia is one of the most common clinical problems. It is often accompanied by skin and soft tissue infections and has a > 50\% mortality rate [16-18]. Atypical manifestations of V. vulnificus infection include pneumonia, meningoencephalitis, peritonitis, and pyogenic spondylitis [9,19,20].

Watery diarrhea, abdominal pain, nausea, vomiting, fever, and chills without skin and soft tissue lesions are the symptoms associated with gastrointestinal tract limited infections caused by V. vulnificus, which may oc- cur in immunocompetent individuals; however, death due to gastrointestinal tract limited infections is rare [5]. Moreover, gastrointestinal symptoms such as nausea, vomiting, and abdominal pain are often preceded by primary septicemia with skin and soft tissue infections $[2,21]$. Therefore, distinguishing between simple gastrointestinal symptoms and premonitory symptoms that can worsen to septicemia is important, especially in individuals at high risk for V. vulnificus infection [22].

Patients with primary septicemia caused by V. vulnificus infection require hospitalization and are typically in septic shock upon admission to the emergency room. Primary septicemia is a bacteremia that usually has no definite route of infection, with the portal of entry presumably either at the small intestine or the proximal colon $[16,23]$. It typically begins with an abrupt fever, chills, and gastrointestinal symptoms; metastatic infection appears as skin lesions that usually occur in the lower extremities with bullae, ecchymosis, or 
maculopapular rash. Skin lesions are aggravated by necrotic ulcer, necrotizing fasciitis or myonecrosis (Fig. 2) $[2,16,24,25]$.

Wound infections differ from primary septicemia because of its portal of entry in the skin; V. vulnificus enters through existing wounds or through areas of traumatic damage. The extent of wound infection with V. vulnificus varies from mild to severe. Wound infection can occur in immunocompetent individuals, such as those with simple gastroenteritis. However, in severe cases, more than half of patients suffer from severe cellulitis accompanied by bullae or ecchymosis within 24 hours of symptom onset. Skin lesions can worsen to necrotizing fasciitis or gangrene. In contrast to primary septicemia, skin lesions are limited to the areas where $\mathrm{V}$. vulnificus is inoculated, and metastatic infections usually do not occur $[2,5,16,24]$.

In a study on V. vulnificus infections in Florida from 1981 to 1987,38 out of 62 patients had primary septicemia, 17 had wound infections, and seven had gastrointestinal illness without septicemia or wound infections [22]. A study of V. vulnificus infections in Taiwan investigated clinical and epidemiologic features from 1995 to 2000; among the 84 total patients, 20 had primary septicemia and 57 had wound infections. A similar previous study in Taiwan found that no patients had gastrointestinal illness $[4,17]$. Most patients with gastroenteritis or diarrheal illness in Taiwan do not seek care at the large teaching hospitals; they also do not usually have a stool culture, which might explain the lack of patients with gastrointestinal illness attributable to V. vulnificus [4,17]. A study on V. vulnificus infections in South Korea from 2000 to 2011 yielded clinical and epidemiological findings similar to the studies in Florida and Taiwan [18]. These three studies show a similar clinical profile, although they were conducted at different times and places (Table 1).

\section{DIAGNOSIS}

V. vulnificus infection is suspected based on clinical and epidemiological findings and is confirmed by bacteriological culture $[16,26]$. Because bacteremia is common, routine blood cultures should be performed when $\mathrm{V}$. vulnificus infection is suspected. Gram stain and cul- ture of a specimen obtained from skin lesions such as abscess or bullae is helpful to rapidly identify bacteria. Stool cultures are occasionally useful, requiring thiosulfate-citrate-bile salts-sucrose agar for isolation [16]. Culture tests using blood or skin lesion samples is the most important method for diagnosing V. vulnificus sepsis. However, since V. vulnificus is highly sensitive to antibiotic administration, it is necessary to check whether any antibiotic has been administered prior to performing the culture test.

Chart analysis of data from 23 patients with V. vulnificus subjected to blood culture tests, which were performed after admission to the researcher's hospital, showed that even though the culture test was performed approximately 24 hours after antibiotic administration in eight patients, not even a single patient tested positive for V. vulnificus. Therefore, if a patient with suspected V. vulnificus sepsis undergoes a culture test after antibiotic administration, V. vulnificus sepsis may not be ruled out even if it is not detected [27].

Microbiological culture provides high specificity, but diagnosis takes a long time. Furthermore, V. vulnificus is susceptible to many antibiotics; therefore, infection in patients previously treated with antibiotics before culture is difficult to diagnose accurately [28]. Polymerase chain reaction (PCR) is also useful for early diagnosis of $V$. vulnificus infection, even in patients previously treated with antibiotics [28]. Using blood samples, the conventional PCR and nested PCR assays showed specificities of $100 \%$ and $73 \%$, respectively. The real-time PCR assay had $100 \%$ sensitivity and specificity as a positive result using a cutoff value of $<38 \mathrm{cp}$ [28]. The real-time PCR assay to detect $V$. vulnificus-specific genes is not only the most sensitive and specific diagnostic method but also the most rapid technique [28]. In a recent study, V. vulnificus DNA copy numbers were higher in tissue samples than in blood samples from V. vulnificus-infected patients, showing that skin lesions are more useful than blood samples for PCR-based diagnosis of V. vulnificus-infected patients [27]. Real-time PCR using serum samples collected from 14 patients at admission showed a median V. vulnificus DNA load of 638.5 copies/mL of blood (interquartile range [IQR], 37 to 3,225), while real-time PCR using the initial tissue specimen at admission showed a median of 16,650 copies/mL of tissue fluid (IQR, 4,419 to 832,500; $p=0.022$ ). Compared 
Table 1. Demographic and clinical features of patients with primary septicemia and wound infections caused by Vibrio vulnificus

\begin{tabular}{|c|c|c|c|}
\hline \multirow{2}{*}{ Variable } & \multicolumn{3}{|c|}{ No. of patients (\%) } \\
\hline & Florida, USA $(\mathrm{n}=55)$ & South Korea $(n=34)$ & Taiwan $(\mathrm{n}=84)$ \\
\hline \multicolumn{4}{|l|}{ Sex } \\
\hline Male & $49(89.1)$ & $30(88.2)$ & $61(72.6)$ \\
\hline Female & $6(10.9)$ & $4(11.8)$ & $23(27 \cdot 4)$ \\
\hline Age, yr & $61.8(23-84)$ & $57 \cdot 3(43-74)$ & $60(9-87)$ \\
\hline \multicolumn{4}{|l|}{ Route of exposure } \\
\hline Consumption of seafood & $35(63.6)$ & $25(73 \cdot 5)$ & $2(3.6)$ \\
\hline Wound exposure to seawater & $15(27 \cdot 3)$ & $5(14.7)$ & $18(32.7)$ \\
\hline Unknown & $5(9.1)$ & $4(11.8)$ & $35(63.6)$ \\
\hline \multicolumn{4}{|l|}{ Underlying condition } \\
\hline Chronic liver disease & $27(49.1)$ & $32(94.1)$ & $45(53.6)$ \\
\hline Chronic alcoholism & & $27(79.4)$ & $15(17.8)$ \\
\hline Hepatitis B or C virus & & $5(14 \cdot 7)$ & $21(25 \cdot 0)$ \\
\hline Other chronic disease without liver disease & $15(27 \cdot 3)$ & 0 & $32(38.1)$ \\
\hline Diabetes mellitus & & & $13(15 \cdot 5)$ \\
\hline Steroid use & & & $10(11.9)$ \\
\hline Renal insufficiency & & & $6(7.1)$ \\
\hline Other malignancies & & & $3(3.6)$ \\
\hline None & $13(23.6)$ & $2(5 \cdot 9)$ & $12(14 \cdot 3)$ \\
\hline \multicolumn{4}{|l|}{ Signs \& symptoms on admission } \\
\hline Any gastrointestinal symptom & $29(52.7)$ & $12(35 \cdot 3)$ & $20(23.8)$ \\
\hline Any skin and soft tissue lesion & $38(69.1)$ & $27(79 \cdot 4)$ & $57(67.9)$ \\
\hline Bacteremia present & $43(78.2)$ & $18(52.9)$ & $7(8.3)$ \\
\hline Surgery performed & $14(25 \cdot 5)$ & $15(44 \cdot 1)$ & $49(58.3)$ \\
\hline Appropriate antibiotics received & $37(67 \cdot 3)$ & $27(79.4)$ & $67(79.8)$ \\
\hline \multicolumn{4}{|l|}{ Outcome } \\
\hline Survived & $30(54 \cdot 5)$ & $18(52.9)$ & $57(67.9)$ \\
\hline Died & $25(45 \cdot 5)$ & $16(47.1)$ & $25(29.8)$ \\
\hline Unknown & 0 & 0 & $2(2.4)$ \\
\hline
\end{tabular}

Values are presented as number (\%) or median (range).

${ }^{a} 55$ patients in Florida, USA, 1981 to 1987 [22] vs. 84 patients in Taiwan 1995 to 2000 [4] vs. 34 patients in South Korea, 2000 to $2011[18]$.

with blood, DNA copies in tissue are less affected by short-term antibiotic administration, and the number of DNA copies actually increases in some patients after antibiotic administration. Therefore, there are typically a larger number of DNA copies in the tissues than in the blood of patients with $\mathrm{V}$. vulnificus infection. In patients who are admitted after receiving antibiotics active against V. vulnificus, a PCR test using skin lesion specimens may be more useful than blood samples.
Since studies have shown that the number of DNA copies is higher in the tissues than in the blood, the actual number of bacteria may be indirectly inferred to be higher in the tissues than in the blood. Thus, the rate of positive results in culture tests may be higher with tissue specimens of the lesion than with blood, which may require further studies [27].

Among the various marine isolates, V. vulnificus and Vibrio cholerae characteristically show resistance against 
polymyxin B and colistin; thus, when susceptibility to colistin is reported, the possibility of false identification of V. vulnificus should be considered [29].

\section{TREATMENT}

Data on 62 cases of V. vulnificus infection reported by Florida health authorities showed that the mortality rate was 33\% when antibiotics were administered within 24 hours after admission, but increased to $63 \%$ and $100 \%$ when antibiotic administration was delayed to 48 to 72 and $>72$ hours post-admission, respectively [22]. These results demonstrate the importance of timely early antibiotic administration.

Case fatality rates for V. vulnificus infections have been shown to increase with delays between the onset of illness and administration of antibiotics. Therefore, if a patient is suspected to be infected with V. vulnificus, appropriate antibiotic treatment should be immediately administered $[2,5,16]$. Furthermore, in many patients with serious skin and soft tissue infections such as necrotizing fasciitis, surgical interventions such as debridement or fasciotomy are necessary in addition to antibiotics treatment to remove necrotic tissue and bacteria $[2,30,31]$. A retrospective study on necrotizing soft-tissue infections including V. vulnificus-related necrotizing fasciitis in 65 patients demonstrated the importance of early surgical intervention based on the time from admission to surgery $(25 \pm 39$ hours in the survival group and $90 \pm 95$ hours in the mortality group) [32].

A retrospective Taiwanese study on V. vulnificus-related necrotizing fasciitis outcomes in 121 patients also reported on the importance of early surgical intervention: the group of patients who received surgical treatment within 12 hours from admission had a significantly lower mortality rate than patients who received similar treatment 12 to 24 and $>24$ hours post-admission [33]. However, since these studies were retrospective, the possibility of selection bias may exist where surgeries were performed on patients with conditions mild enough to actually undergo surgery. Therefore, future prospective case-control studies are needed.

V. vulnificus is sensitive to most antibiotics in vitro, except for colistin; antibiotics that are effective against V. vulnificus in vitro are third-generation cephalosporins (ceftriaxone, cefotaxime, or ceftazidime), piperacillin-tazobactam, carbapenems (imipenem or meropenem), tetracyclines (doxycycline or tetracycline), aminoglycoside (gentamicin or amikacin), fluoroquinolones (ciprofloxacin, levofloxacin, or moxifloxacin), and sulfamethoxazole-trimethoprime [31,34]. In most in vitro or in vivo mouse model experiments on antibiotic susceptibility, tetracyclines are reported to have significantly higher efficacy than penicillin or cephalosporins. This has been attributed to the fact that the tetracycline class of antibiotics achieves better penetration into tissues with poor perfusion when V. vulnificus infection occurs, and has inhibitory effects against the synthesis of proteins, such as various toxins and enzymes produced by V. vulnificus [35].

A time-kill study on V. vulnificus demonstrated that cefotaxime and minocycline have synergistic effects, and a V. vulnificus mouse model experiment also reported that the combined administration of the two drugs showed statistically significant effects $(p<0.01)$ [36].

A study on the efficacy of quinolone also confirmed that it was as effective as cefotaxime and minocycline; quinolone was reported to be effective in mouse models, as its concentration was sufficiently maintained in the necrotized interstitial fluid or blister and thrombosis caused by V. vulnificus sepsis [37].

A time-kill study on ciprofloxacin and cefotaxime confirmed their synergistic action, while an animal experiment confirmed that the combined administration of cefotaxime and ciprofloxacin offered greater survival benefits than a combination of cefotaxime and minocycline or cefotaxime alone [38,39].

The CDC recommends doxycycline $100 \mathrm{mg}$ intravenously or orally twice a day plus ceftazidime (or any other third-generation cephalosporin) 1 to $2 \mathrm{~g}$ intravenously every 8 hours for the treatment of $V$. vulnificus infection $[5,16,40]$. In a retrospective study on $89 \mathrm{~V}$. vulnificus-infected patients, a third-generation cephalosporin plus minocycline or ciprofloxacin with or without minocycline was found to be more effective than a third-generation cephalosporin alone [41]. An animal experiment suggested that the combination of ciprofloxacin and cefotaxime is an effective treatment option for V. vulnificus-infected people [39]. A recent study suggested that cefepime combined with doxycycline or ciprofloxacin should be initiated to cover gram-nega- 
tive-resistant organisms and V. vulnificus while awaiting a microbiological diagnosis; once a diagnosis of V. vulnificus septicemia is confirmed, treatment can be safely changed to ceftriaxone combined with doxycycline or ciprofloxacin [42]. Another recent study suggested that tigecycline alone might be a better therapeutic option than a traditional combination regimen of ceftazidime plus doxycycline in patients with V. vulnificus infection $[43,44]$.

\section{PROGNOSIS}

V. vulnificus infections are life-threatening, and favorable prognosis depends on early diagnosis and appropriate treatment. Several previous studies suggested a marker for predicting prognosis among patients with V. vulnificus infection. Acute Physiology And Chronic Health Evaluation II (APACHE II) and Mortality in Emergency Department Sepsis (MEDS) scores at hospital admission were suggested as useful prognostic indicators in primary septicemia or wound infections caused by V. vulnificus [31,45,46]. In another study, the level of V. vulnificus DNA loads were significantly correlated with mortality [47].

The median DNA copy upon admission was 2,300 copies/mL of blood (IQR, 558 to 11,350) in patients who died of V. vulnificus sepsis, which was significantly higher than 316.5 copies/mL of blood (IQR, 51 to 705) in those who survived $(p=0.011)$. When a patient is admitted for V. vulnificus sepsis, using real-time PCR for the identification of V. vulnificus DNA copies in the serum as an early prognostic indicator of mortality may be clinically useful [47].

The level of V. vulnificus DNA was shown to be substantially higher in nonsurvivors than in survivors of V. vulnificus septicemia, and the V. vulnificus DNA load correlated with the APACHE II score $[18,47]$. Notably, elevated tumor necrosis factor $\alpha$ (TNF- $\alpha$ ) levels were found in patients with V. vulnificus sepsis compared to those in healthy individuals. The group of patients who died of $V$. vulnificus sepsis showed a median TNF- $\alpha$ level of $261.0 \mathrm{pg} / \mathrm{mL}$ (IQR, 101.0 to 376.0), which was significantly higher than $69.5 \mathrm{pg} / \mathrm{mL}$ (IQR, 17.5 to 103.5) in those who survived $(p=0.001)$. Such a difference was also found in the blood test results, which were evaluated 6 to 48 hours post-admission and antibiotic administration; the mortality group showed a significantly elevated TNF- $\alpha$ level $(p=0.044)$. This confirmed that checking the serum TNF- $\alpha$ level upon admission as an early prognostic factor of mortality may be clinically useful [48]. In a retrospective study in South Korea, approximately half of the patients with $\mathrm{V}$. vulnificus infection died $\leq 72$ hours after hospital admission [18]. In this study, patient survival differed significantly based on $\mathrm{pH}$ level at hospital admission; in predicting death, an optimal cutoff $\mathrm{pH}$ level of $<7.35$ had a sensitivity of $100 \%$ and specificity of $83 \%$ [17]. This study showed that the initial $\mathrm{pH}$ level of patients was the simplest and the most accurate predictive marker of death [18].

\section{CONCLUSIONS}

V. vulnificus is capable of causing illness ranging from mild gastrointestinal tract-limited infections to severe septicemia with necrotizing fasciitis. Patients with a presumptive diagnosis of V. vulnificus infection should be immediately started on antibiotics therapy and surgical interventions if needed. The recommended antibiotics treatment regimen is ceftriaxone plus doxycycline or ceftriaxone plus ciprofloxacin. $\mathrm{pH}$ level upon admission, APACHE II score, and V. vulnificus DNA load can help predict the prognosis for patients with $\mathrm{V}$. vulnificus infection.

\section{Conflict of interest}

No potential conflict of interest relevant to this article was reported.

\section{Acknowledgments}

This study was supported by research funds (2015) from Chosun University.

\section{REFERENCES}

1. Centers for Disease Control and Prevention. Summary of human Vibrio isolates reported to CDC, 2004 [Internet]. Atlanta (GA): CDC, 2004 [cited 2018 May 21]. Available from: https://www.cdc.gov/nationalsurveillance/pdfs/ vibrio2004.pdf. 
2. Bross MH, Soch K, Morales R, Mitchell RB. Vibrio vulnificus infection: diagnosis and treatment. Am Fam Physician 2007;76:539-544.

3. Centers for Disease Control and Prevention (CDC). Preliminary FoodNet data on the incidence of infection with pathogens transmitted commonly through food: 10 States, United States, 2005. MMWR Morb Mortal Wkly Rep 2006;55:392-395.

4. Hsueh PR, Lin CY, Tang HJ, et al. Vibrio vulnificus in Taiwan. Emerg Infect Dis 2004;10:1363-1368.

5. Daniels NA. Vibrio vulnificus oysters: pearls and perils. Clin Infect Dis 2011;52:788-792.

6. Johnston JM, Becker SF, McFarland LM. Vibrio vulnificus. Man and the sea. JAMA 1985;253:2850-2853.

7. Barton JC, Acton RT. Hemochromatosis and Vibrio vulnificus wound infections. J Clin Gastroenterol 2009;43: 890-893.

8. Haq SM, Dayal HH. Chronic liver disease and consumption of raw oysters: a potentially lethal combination: a review of Vibrio vulnificus septicemia. Am J Gastroenterol 2005;100:1195-1199.

9. Park SD, Lee JY, Kim HD, Yoon NH. Clinical study of Vibrio vulnificus sepsis. Korean J Dermatol 2006;44:696707.

10. Wright AC, Simpson LM, Oliver JD. Role of iron in the pathogenesis of Vibrio vulnificus infections. Infect Immun 1981;34:503-507.

11. Lesic B, Foulon J, Carniel E. Comparison of the effects of deferiprone versus deferoxamine on growth and virulence of Yersinia enterocolitica. Antimicrob Agents Chemother 2002;46:1741-1745.

12. Neupane GP, Kim DM. Comparison of the effects of deferasirox, deferiprone, and deferoxamine on the growth and virulence of Vibrio vulnificus. Transfusion 2009;49:1762-1769.

13. Lee SH, Chung BH, Lee WC. Retrospective analysis of epidemiological aspects of Vibrio vulnificus infections in Korea in 2001-2010. Jpn J Infect Dis 2013;66:331-333.

14. Shin YH, Lee JK, Oh KS, et al. National surveillance of Vibrio vulnificus infections in 1998. Korean J Infect Dis 1999;31:232-236.

15. Merkel SM, Alexander S, Zufall E, Oliver JD, HuetHudson YM. Essential role for estrogen in protection against Vibrio vulnificus-induced endotoxic shock. Infect Immun 2001;69:6119-6122.

16. Kim DM, Hong SJ. Vibrio vulnificus sepsis. Korean J Med
2012;82:671-679.

17. Chuang YC, Yuan CY, Liu CY, Lan CK, Huang AH. Vibrio vulnificus infection in Taiwan: report of 28 cases and review of clinical manifestations and treatment. Clin Infect Dis 1992;15:271-276.

18. Yun NR, Kim DM, Lee J, Han MA. pH level as a marker for predicting death among patients with Vibrio vulnificus infection, South Korea, 2000-2011. Emerg Infect Dis 2015;21:259-264.

19. Ok HS, Kim BK, Kim KH, et al. Vertebral osteomyelitis resulting from hematogenous spread of Vibrio vulnificus gastroenteritis. Korean J Med 2014;86:519-522.

20. Kim CS, Bae EH, Ma SK, Kim SW. Severe septicemia, necrotizing fasciitis, and peritonitis due to Vibrio vulnificus in a patient undergoing continuous ambulatory peritoneal dialysis: a case report. BMC Infect Dis 2015;15:422.

21. Shapiro RL, Altekruse S, Hutwagner L, et al. The role of Gulf Coast oysters harvested in warmer months in Vibrio vulnificus infections in the United States, 1988-1996. Vibrio Working Group. J Infect Dis 1998;178:752-759.

22. Klontz KC, Lieb S, Schreiber M, Janowski HT, Baldy LM, Gunn RA. Syndromes of Vibrio vulnificus infections. Clinical and epidemiologic features in Florida cases, 19811987. Ann Intern Med 1988;109:318-323.

23. Chen Y, Satoh T, Tokunaga O. Vibrio vulnificus infection in patients with liver disease: report of five autopsy cases. Virchows Arch 2002;441:88-92.

24. Dechet AM, Yu PA, Koram N, Painter J. Nonfoodborne Vibrio infections: an important cause of morbidity and mortality in the United States, 1997-2006. Clin Infect Dis 2008;46:970-976.

25. Tacket CO, Brenner F, Blake PA. Clinical features and an epidemiological study of Vibrio vulnificus infections. J Infect Dis 1984;149:558-561.

26. Ma KA, Lee SM, Kim MS, et al. Emergency debridement with empirical antibiotics treatment for clinically diagnosed Vibrio sepsis. Korean J Infect Dis 1997;29:297303.

27. Lee JY, Kim SW, Kim DM, Yun NR, Kim CM, Lee SH. Clinical usefulness of real-time polymerase chain reaction for the diagnosis of Vibrio vulnificus infection using skin and soft tissues. Am J Trop Med Hyg 2017;97:443-446.

28. Kim HS, Kim DM, Neupane GP, et al. Comparison of conventional, nested, and real-time PCR assays for rapid and accurate detection of Vibrio vulnificus. J Clin Microbiol 2008;46:2992-2998. 
29. Ryu HH, Lee JY, Yun NR, Kim DM. Necrotizing soft tissue infection with gas formation caused by Vibrio vulnificus and misdiagnosed as Pseudomonas aeruginosa. Am J Emerg Med 2013;31:464.

30. Halow KD, Harner RC, Fontenelle LJ. Primary skin infections secondary to Vibrio vulnificus: the role of operative intervention. J Am Coll Surg 1996;183:329-334.

31. Bilton BD, Zibari GB, McMillan RW, Aultman DF, Dunn G, McDonald JC. Aggressive surgical management of necrotizing fasciitis serves to decrease mortality: a retrospective study. Am Surg 1998;64:397-400.

32. McHenry CR, Piotrowski JJ, Petrinic D, Malangoni MA. Determinants of mortality for necrotizing soft-tissue infections. Ann Surg 1995;221:558-563.

33. Chao WN, Tsai CF, Chang HR, et al. Impact of timing of surgery on outcome of Vibrio vulnificus-related necrotizing fasciitis. Am J Surg 2013;206:32-39.

34. Han F, Walker RD, Janes ME, Prinyawiwatkul W, Ge B. Antimicrobial susceptibilities of Vibrio parahaemolyticus and Vibrio vulnificus isolates from Louisiana Gulf and retail raw oysters. Appl Environ Microbiol 2007;73:70967098.

35. Bowdre JH, Hull JH, Cocchetto DM. Antibiotic efficacy against Vibrio vulnificus in the mouse: superiority of tetracycline. J Pharmacol Exp Ther 1983;225:595-598.

36. Chuang YC, Liu JW, Ko WC, Lin KY, Wu JJ, Huang KY. In vitro synergism between cefotaxime and minocycline against Vibrio vulnificus. Antimicrob Agents Chemother 1997;41:2214-2217.

37. Tang HJ, Chang MC, Ko WC, Huang KY, Lee CL, Chuang YC. In vitro and in vivo activities of newer fluoroquinolones against Vibrio vulnificus. Antimicrob Agents Chemother 2002;46:3580-3584.

38. Kim DM, Lym Y, Jang SJ, et al. In vitro efficacy of the combination of ciprofloxacin and cefotaxime against Vibrio vulnificus. Antimicrob Agents Chemother
2005;49:3489-3491.

39. Jang HC, Choi SM, Kim HK, et al. In vivo efficacy of the combination of ciprofloxacin and cefotaxime against Vibrio vulnificus sepsis. PLoS One 2014;9:e101118.

40. Centers for Disease Control and Prevention. Vibrio vulnificus: general information [Internet]. Atlanta (GA): CDC, 2018 [cited 2018 May 21]. Available from: https:// www.cdc.gov.

41. Chen SC, Lee YT, Tsai SJ, et al. Antibiotic therapy for necrotizing fasciitis caused by Vibrio vulnificus: retrospective analysis of an 8 year period. J Antimicrob Chemother 2012;67:488-493.

42. Trinh SA, Gavin HE, Satchell KJF. Efficacy of ceftriaxone, cefepime, doxycycline, ciprofloxacin, and combination therapy for Vibrio vulnificus foodborne septicemia. Antimicrob Agents Chemother 2017;61:e01106-17.

43. Yu W, Shen X, Pan H, Xiao T, Shen P, Xiao Y. Clinical features and treatment of patients with Vibrio vulnificus infection. Int J Infect Dis 2017;59:1-6.

44. Lin YS, Hung MH, Chen CC, Huang KF, Ko WC, Tang HJ. Tigecycline salvage therapy for necrotizing fasciitis caused by Vibrio vulnificus: case report in a child. J Microbiol Immunol Infect 2016;49:138-141.

45. Kuo Chou TN, Chao WN, Yang C, Wong RH, Ueng KC, Chen SC. Predictors of mortality in skin and soft-tissue infections caused by Vibrio vulnificus. World J Surg 2010;34:1669-1675.

46. Chen SC, Chan KS, Chao WN, et al. Clinical outcomes and prognostic factors for patients with Vibrio vulnificus infections requiring intensive care: a 10-yr retrospective study. Crit Care Med 2010;38:1984-1990.

47. Kim DM, Jung SI, Jang HC, et al. Vibrio vulnificus DNA load and mortality. J Clin Microbiol 2011;49:413-415.

48. Lee JY, Kim DM, Yun NR, et al. Tumor necrosis factor- $\alpha$ and mortality in patients infected with Vibrio vulnificus. Am J Trop Med Hyg 2011;84:426-428. 